\title{
Risques naturels, expertise et situation de crise
}

Michel Campillo ${ }^{(1)}$ (michel.campillo@ujf-grenoble.fr), Christophe Voisin ${ }^{(1)}$ et Yves Nicolas ${ }^{(2)}$ (1) Institut des Sciences de la Terre, Université Joseph Fourier et CNRS, BP 53, 38041 Grenoble Cedex 9 (2) Institut de la communication et des médias (ICM), Université Stendhal, Grenoble

Nous vivons dans une période où la parole des scientifiques est questionnée dans le public. En particulier, le rôle d'expert semble de plus en plus difficile à tenir par les scientifiques face à l'explosion des moyens de communication immédiats.

Les risques naturels sont un

bon exemple des difficultés rencontrées par les experts.

Nous discutons dans cet article les suites judiciaires de l'affaire du séisme de L'Aquila, survenu en avril 2009, dans laquelle les plaignants ont reproché à des géophysiciens italiens (condamnés en première instance, puis acquittés en appel) d'avoir, par une déclaration rassurante, laissé penser que le risque sismique était négligeable.
Les sciences ont créé des représentations qui permettent de comprendre et de quantifier des processus dont les occurrences ne sont décrites que par des probabilités. La communication de ces résultats reste très difficile pour un public non averti. Les représentations probabilistes ont pourtant des applications qui, loin des laboratoires, touchent directement le grand public. Un exemple en est apporté par les aléas naturels.

Un aléa naturel est le résultat d'interactions complexes, non linéaires, de systèmes physiques mal connus dont la dynamique relève d'échelles de temps et d'espace imbriquées. Pour cette raison, la science actuelle ne répond pas directement à des questions comme la prévision à court terme des séismes. Elle tente, par contre, de quantifier des probabilités d'occurrence, des niveaux d'aléa relatifs, des conséquences potentielles de scénarios probables, tous éléments de nature à éclairer la décision politique pour l'aménagement du territoire, la mise en ouvre de technologies potentiellement dangereuses, l'aide aux populations, etc. La science ne peut néanmoins pas apporter de réponses déterministes qui permettraient d'établir des éléments d'aide à la décision qui soient définitifs et simples à exprimer, car chacun de ces éléments est empreint d'une très grande incertitude. Si les frontières et les procédures de l'activité de recherche scientifique sont assez bien définies et comprises, les chercheurs sont souvent appelés à sortir de ce cadre pour jouer un rôle d'expert à la demande d'opérateurs économiques ou politiques. Cette position d'expert amène le scientifique dans un champ d'activité différent de celui de la recherche proprement dite.

Nous traiterons ici des séismes, qui se caractérisent par de hauts niveaux d'incertitude, voire de controverse scientifique, et des enjeux sociaux très importants. Les exemples récents des séismes d'Haiti (avec le chiffre de 200000 victimes) et du Japon (qui a montré, avec la centrale de Fukushima, la vulnérabilité d'un pays à très haut niveau de développement technique) ont rappelé, une fois de plus, l'impact des événements naturels. Participer aux efforts d'information et de préparation, et éventuellement apporter des éléments scientifiques d'aide à la décision en situation de crise, sont (a priori) des activités qui font partie des devoirs d'un scientifique responsable. Dans ce contexte, l'attention de la communauté scientifique internationale a été attirée par la mise en cause judiciaire de géophysiciens italiens dans l'affaire dite de L'Aquila, suite au séisme qui a affecté cette ville le 6 avril 2009, faisant 308 morts et détruisant plusieurs milliers de bâtiments.

Nous utiliserons cet exemple pour illustrer les mécanismes qui rendent difficile cet exercice d'expertise. Nous présenterons rapidement les recommandations qui ont été faites par le Comité d'éthique du CNRS, à la suite de cette affaire. 


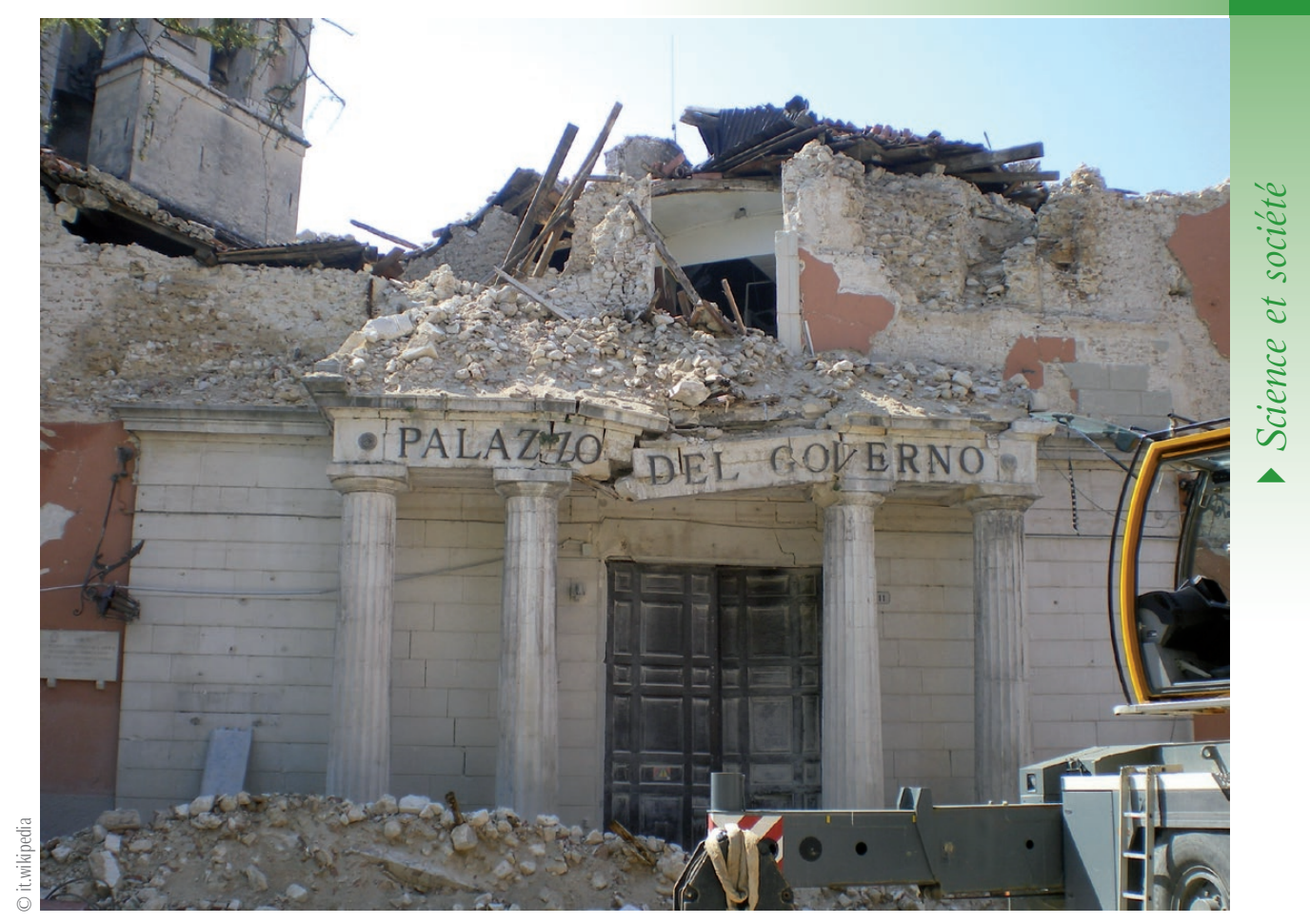

La préfecture de L'Aquila après le séisme, un symbole de la destruction.

\section{L'affaire du séisme de L'Aquila}

Donnons quelques éléments de contexte à l'exemple de l'Aquila. L'émotion a été créée par la lourde condamnation (le 22 octobre 2012), dont six ans d'emprisonnement en première instance, de participants à une réunion de type commission d'experts. Les personnalités incriminées étaient pour la plupart des chercheurs reconnus dans leur domaine. Ces mêmes personnes ont été acquittées le 10 novembre 2014, lors $\mathrm{du}$ procès en appel. Nous essayerons de décrire l'engrenage qui a conduit à cette condamnation en première instance.

Notons que le contexte politique dans lequel se produit la mise en cause des scientifiques a été utilisé comme élément d'explication (e.g. [1]). Des années de contraintes budgétaires et de pressions ont probablement pu peser sur les possibilités d'indépendance des universitaires italiens soumis à des attributions de crédit par des structures gouvernementales, et une partie de l'opinion a voulu aussi faire le procès du système de l'ère Berlusconi. Ces éléments spécifiques ne sont pas discutés ici, pas plus que ceux du débat judiciaire, mais il faut noter que les contextes de pression sont toujours présents, à des niveaux variables bien sûr, et il serait naïf de penser que cette dimension soit totalement absente dans d'autres contextes.

\section{Séisme de L'Aquila : les faits}

Commençons par quelques éléments factuels. L'Aquila se trouve dans la région des Abruzzes, une des zones sismiquement les plus actives d'Italie et d'Europe [2]. Le séisme de 2009 correspond à un événement dont la probabilité avait été évaluée comme grande avant même son occurrence (cf. tableau 1). Pour être plus précis, le niveau des sollicitations sismiques (accélérations du sol) a correspondu aux documents produits par les scientifiques italiens. Il n'y donc pas eu de défaut d'évaluation ou de communication du risque à moyen terme. Pour bien comprendre la spécificité des événements, il faut prendre en compte plusieurs éléments. Le premier est la crise sismique qui a précédé le séisme : des centaines de séismes de magnitudes faibles, mais suffisantes pour qu'ils soient ressentis par la population, se sont produits à partir de décembre 2008. Dans un contexte de légitime inquiétude, le plus fort de ces événements, avec une magnitude de 4, s'est produit seulement sept jours avant le choc principal. La réunion incriminée s'est déroulée, elle, entre ces deux séismes. Nous remarquons ici que le risque d'un fort séisme était déjà discuté dans l'arène publique avant même la réunion du groupe d'experts, et c'est ce qui va la justifier.

Un autre élément factuel qui doit être considéré est la "prévision " d'un fort séisme, qui a été publiée par un ingénieur agissant indépendamment des structures

Tableau 1. Ordre de grandeur des probabilités d'occurrence pour un séisme comme celui de L'Aquila. (Source : processoaquila.wordpress.com).

\begin{tabular}{|l|c|}
\hline $\begin{array}{l}\text { Nombre de séquences sismiques observées en Italie } \\
\text { durant la période 2008-2010. } \\
\text { Une seule séquence a conduit à un séisme de magnitude } M>5,0 \text { (L'Aquila). }\end{array}$ & 127 \\
\hline $\begin{array}{l}\text { Probabilité journalière d'occurrence d'un séisme M > 5,5 } \\
\text { une semaine après un séisme de magnitude 4, } \\
\text { comme celui du } 30 \text { mars } 2009 \text { (modèle ETAS). }\end{array}$ & $510^{-4}$ \\
\hline \begin{tabular}{l} 
Probabilité de gagner le gros lot de la Loterie nationale. \\
\hline
\end{tabular} & $5,210^{-8}$ \\
\hline
\end{tabular}




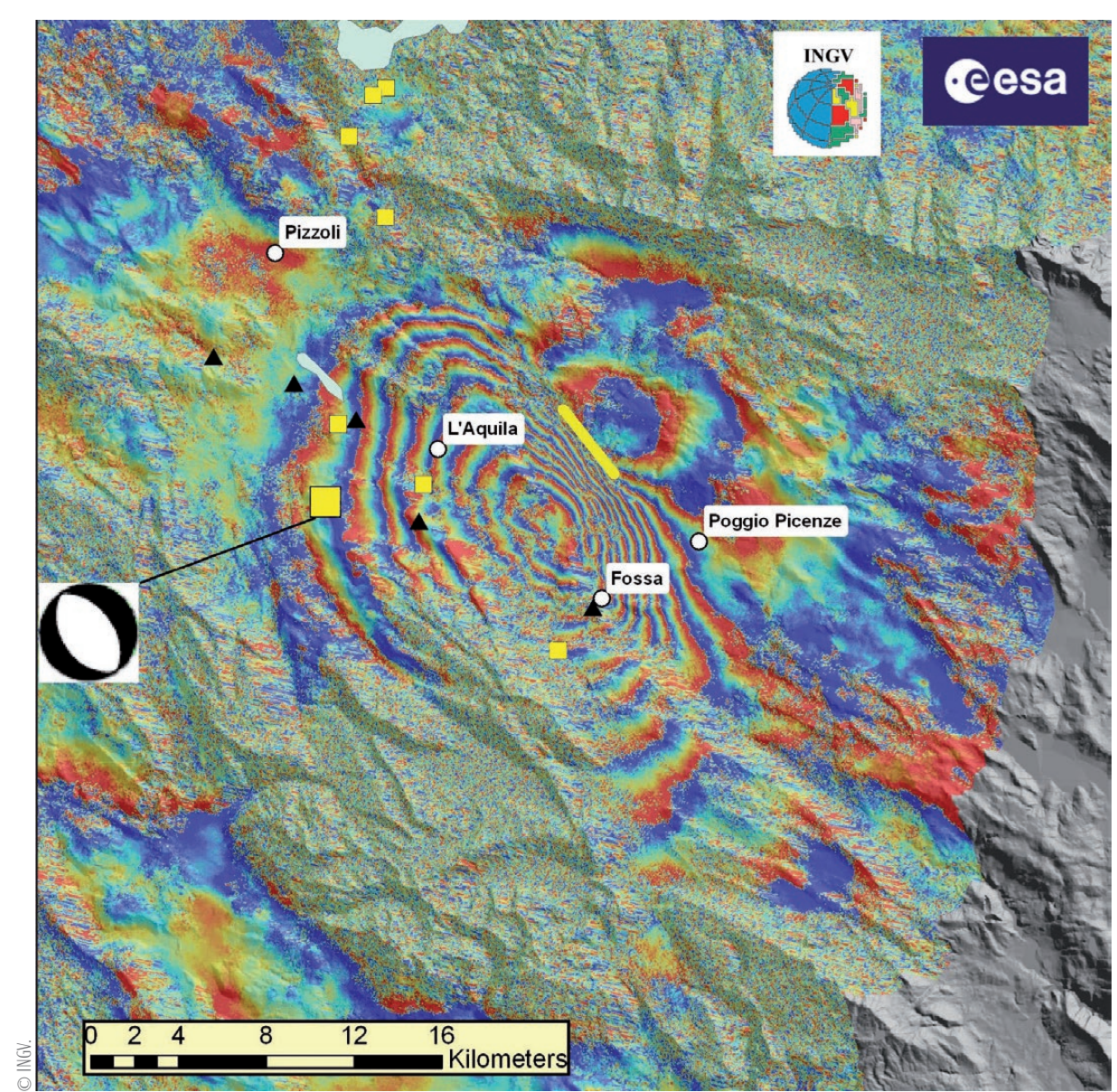

1. Interférogramme de la région de L’Aquila, obtenu par le radar SAR du satellite ENVISAT de l'ESA et interprété par l'Institut italien de géophysique et vulcanologie (INGV). On y voit des franges de couleur indiquant des modifications d'altitude de l'ordre du centimètre, résultant du séisme du 6 avril 2009. Le grand carré jaune désigne le choc principal, de magnitude 6,3. Les plus petits carrés jaunes indiquent les chocs ultérieurs de magnitude supérieure à 5 , et les triangles noirs les stations GPS utilisées pour valider les observations de SAR. La ligne jaune à l'est de L'Aquila représente une fracture de 4 km de long.

\section{〉>}

en charge de l'évaluation du risque sismique. Cette prévision concernait une localité située à $80 \mathrm{~km}$ de l'Aquila, mais a eu un retentissement médiatique et a pu augmenter le niveau de tension dans la population. Elle a été faite sur la base de mesures d'émanations de radon. L'idée de réaliser le suivi temporel du radon trouve des fondements physiques. En effet, le gaz radon est un produit des chaines de désintégration de l'uranium et du thorium, présents naturellement dans les roches. Le radon 222, issu de la désintégration de l'uranium 238, a une demi-vie d'environ quatre jours, et il a été proposé que son transport soit facilité par l'endommagement (ouverture de fissures) associé à la déformation des roches. Si une déformation significative des massifs rocheux près de la surface se produit juste avant les séismes, il serait possible qu'une anomalie
Rischi). La réunion a été organisée très rapidement par la Protection Civile. Elle associera des personnes extérieures et ne durera que peu de temps. À l'issue de la réunion, un représentant de la Protection Civile fait une déclaration rassurante, affirmant que la sismicité en cours est plutôt un élément positif. Les plaignants affirmeront que c'est à la suite de cette déclaration que des personnes qui avaient quitté - ou décidé de quitter - L'Aquila de peur d'un séisme, ont finalement été présentes lors de la secousse du 6 avril et y ont perdu la vie.

Les scientifiques présents à la réunion (sans considération de leur appartenance à la Commission) sont accusés de ne pas avoir informé correctement la population, l'accusation étant que ce défaut de communication aurait été la cause de la mort de certaines victimes. Les plaignants, lors de leurs interventions dans la presse, ne mettent pas en cause l'incapacité réelle de prévoir l'occurrence du séisme et se défendent de vouloir faire le procès de la science. L'affaire judiciaire a été rejugée en appel, et les scientifiques incriminés en première instance ont été acquittés. Nous ne discuterons pas ici les fondements des jugements précédents. Nous voulons par contre comprendre comment nos collègues ont pu se trouver dans cette situation et décrire la nature du piège dans lequel nombre de chercheurs auraient pu tomber.

\section{Une position délicate pour les scientifiques}

Nous pensons en effet que cette affaire trouve son origine dans les conditions dans lesquelles l'expertise a été menée, et il est important à l'avenir que la procédure soit adaptée aux conditions spécifiques, tout en répondant aux exigences éthiques de l'implication des chercheurs dans des activités d'expertise comme celles mises en cause. C'est ce qui a justifié l'autosaisine du Comité d'éthique du CNRS (COMETS), qui a publié un avis sur cette question (www.cnrs.fr/comets/spip.php?article85). Dans son texte, le COMETS rappelle que des chartes de l'expertise existent (charte nationale de l'expertise, charte de l'expertise du CNRS...) et offrent des bases solides d'engagement qui, malheureusement, par leur complexité, sont peu adaptées aux situations de crise où les demandes de recommandations ont un caractère d'urgence. Une réunion décidée hâtivement, comme celle incriminée dans l'affaire de L'Aquila, 
ne satisfait pas aux conditions spécifiées dans les chartes, et on peut imaginer une situation comparable dans beaucoup de pays sismiques. Il est néanmoins impossible pour les scientifiques de décider individuellement de ne pas répondre à des demandes de ce type. On imagine bien que ceux qui auraient refusé de participer à une telle réunion suivie d'une catastrophe seraient à leur tour accusés de ne pas avoir fourni en temps utile les informations nécessaires.

Face à un risque imminent, les principes exprimés dans les chartes générales sont difficiles à prendre en compte, dans la mesure où le commanditaire doit prendre des décisions rapides alors qu'il n'y a pas de réponse simple aux questions posées. La pertinence d'une évaluation probabiliste d'un niveau de risque peut sembler contredite par les faits (occurrence d'un événement de faible probabilité) pour un public non préparé (cf. tableau 1). Il serait rassurant de penser qu'il est possible de démontrer qu'un avis s'est bien appuyé sur une méthode scientifique rigoureuse. Néanmoins, on peut légitimement craindre que dans beaucoup de domaines liés aux risques naturels, existent à la fois des incertitudes fondamentales et des controverses qui animent le débat scientifique, dont la communication est difficile. L'expertise devra mettre au jour les méthodes et les interprétations, même diverses, qui font l'objet d'un consensus suffisant dans la communauté scientifique. Ces controverses nourrissent aussi dans l'espace public des débats d'opinion, qui échappent aux procédés auxquels les scientifiques sont habitués. C'est une dimension qui ne peut pas être ignorée. Les scientifiques impliqués dans ces expertises devront se préparer à voir la forme d'autorité d'un savoir construit être mise en doute par les convictions diverses qui s'expriment dans l'espace public.

\section{Les suites du débat juridique}

Beaucoup de voix se sont élevées pour attaquer le bien fondé des lourdes condamnations qui ont touché indifféremment agents opérationnels et scientifiques présents lors de la réunion incriminée, membres ou non de la Commission des grands risques. Nous ne commenterons pas des éléments de ce procès, ni ceux du jugement en appel. $\mathrm{Au}$-delà des réactions que le jugement en première instance a pu provoquer, les décisions d'un juge européen, même discutées en appel, ne peuvent pas être ignorées.
La question de la causalité entre l'action de la commission et les conséquences fatales du séisme ouvre des perspectives d'ordre général. En particulier, l'interprétation donnée par le juge conduit à s'interroger sur l'existence d'une perception nouvelle du statut de la communication scientifique et des responsabilités du scientifique. Ce que prétendrait ce jugement est que l'on pourrait mourir d'un possible défaut de communication. Que celui qui communique " $\mathrm{mal}$ » est donc directement responsable, parce qu'on lui a donné un statut particulier, même s'il ne l'a pas choisi. Que la causalité serait là. Une telle interprétation donne tout son poids au fait social, au détriment du phénomène physique. En participant à des processus d'expertise sur des sujets avec de fortes implications sociales immédiates, les scientifiques doivent être conscients qu'ils s'engagent sur un terrain très éloigné de celui de leurs compétences réelles, et où la pertinence scientifique ne constitue pas une valeur en soi.

\section{Recommandations de bonne conduite :}

comité d'éthique du CNRS. www.cnrs.fr/comets/spip.php?article85

- En s'appuyant sur les chartes existantes, et pour chaque type de risque, des procédures d'engagement dans l'expertise doivent être préparées en avance des situations de crise. La communication est particulièrement difficile sur des phénomènes complexes dont l'évolution requiert des approches probabilistes. Des porte-parole doivent être désignés, préparés et formés pour cette tâche, car l'espace public démocratique requiert d'autres règles et un autre registre que la discussion scientifique.

- L'exploitation des conclusions de l'expertise n'appartient pas à l'expert scientifique, mais au décideur politique. Cela doit être précisé pour le public dans tous les éléments de communication. Les scientifiques n'ont pas à être solidaires des décisions prises à partir d'un ensemble d'informations dont leur expertise n'est qu'un élément.

- L'expert conserve un pouvoir de contrôle sur le contenu de son expertise, qui ne doit pas être dénaturé dans sa présentation au public. Hormis le droit de rectification, l'expert doit bénéficier du régime général prévu par la loi n²013-316 du 16 avril 2013 relative à I'indépendance de l'expertise en matière de santé et d'environnement et à la protection des lanceurs d'alerte. En application de l'article 2 de cette loi, il incombe également aux organismes d'informer la Commission de déontologie instaurée par ce texte sur les actions qu'ils entreprennent en matière d'expertise, et notamment sur les codes de déontologie qu'ils mettent en place.

- Le contenu scientifique de l'expertise inclut un exposé des informations techniques et de l'état de la connaissance. Ce contenu doit être rendu public rapidement. Les experts ne doivent pas être aveugles vis-à-vis des débats d'opinion en cours dans la sphère publique. Dans les problèmes d'aléas naturels, les conclusions scientifiques sont souvent entachées de fortes incertitudes liées à la dynamique du système considéré. La communication doit tenter de rendre cet état de fait compréhensible par tous.

\section{Références}

1 D.E. Alexander, Natural Hazards 72 (2014) 1159-1173.

2 D. Giardini et al., "SHARE European Seismic Hazard Map, 10\% Exceedance Probabilities in 50 years", doi 10.2777/30345, ISBN-13, 978-92-79-25148-1. Carte des risques sismiques : www.share-eu.org

3• M. Campillo et Y. Nicolas, « La prévision des tremblements de terre : un dialogue à risque aux frontières de la science ", Le temps des savoirs 2 (2000) 39-60. 